\title{
Forme du perçu, structure du langage : Merleau-Ponty avec et contre Saussure
}

Par JEANNE-MARIE ROUX

Université Paris 1 Panthéon-Sorbonne

\section{Introduction. Deux voies pour la phénoménologie?}

$\mathrm{Au} \S 124$ des Idées directrices pour une phénoménologie transcendantale, Edmund Husserl introduit «les actes expressifs » dans l'architecture phénoménologique qu'il a déployée jusque-là et leur réserve le terme de « signification », afin de conserver le mot «sens» pour désigner le noème en général ${ }^{1}$. Il explicite alors le rôle méthodologique crucial que doit jouer l'étude de ces actes pour toute phénoménologie soucieuse de son «fondement $\gg$ :

Les phénomènes qui répondent aux termes signifier et signification soulèvent des problèmes extraordinairement difficiles ${ }^{2}$. Comme toute science est amenée par sa structure théorique, par tous les traits qui en elle sont de l'ordre de la «doctrine » (théorème, preuve, théorie), à s'objectiver dans un medium

\footnotetext{
${ }^{1}$ La réflexion qui se trouve développée dans ces pages reprend en grande partie des éléments développés dans la deuxième partie de notre thèse de doctorat, Les degrés $d u$ silence. De la juste place du sens dans le langage et dans la perception chez Austin et Merleau-Ponty, rédigée sous la direction de M. Jocelyn Benoist, et soutenue le 26 novembre 2015 à l'Université Paris 1 Panthéon-Sorbonne. Nous sommes heureux de pouvoir chaleureusement remercier ici Charlotte Gauvry et Bruno Leclercq, toute l'équipe «Phénoménologies » de l'Université de Liège, ainsi que les participants du passionnant séminaire "Phénoménologie et grammaire », qui fut l'occasion de l'intervention dont cet article est la trace.

2 «Comme on le voit par le t. II des Études logiques, où ils forment un thème central. » [Note du texte original. Les Études logiques désignent ce que l'on nomme aujourd'hui les Recherches logiques].
} 
spécifiquement « logique », dans le medium de l'expression, les problèmes de l'expression et de la signification sont les premiers que rencontrent les philosophes et les psychologues soucieux de logique générale, et ce sont ensuite les premiers qui exigent une investigation éidétique d'ordre phénoménologique dès qu'on cherche sérieusement à atteindre leur fondement ${ }^{1}$.

Dans ces quelques lignes, Husserl indique clairement le statut de prérequis, de préliminaire indispensable que constitue le fait d'étudier « les problèmes de l'expression et de la signification » si l'on veut fonder la méthode phénoménologique. Mais il complète immédiatement son propos par une note, tout à la fois significative et troublante, dès lors qu'elle suggère qu'une autre voie phénoménologique est possible et donc qu'il est possible, soit de fonder la phénoménologie autrement, soit de renoncer à commencer par la fonder :

En fait, c'était la voie par laquelle les Études logiques tentaient de pénétrer dans la phénoménologie. Une seconde voie partant du côté opposé, celui de l'expérience et des données sensibles, que l'auteur a également suivie depuis le début des années 90 , n'a pas trouvé sa pleine expression dans cette œuvre ${ }^{2}$.

Cet article part du constat que Maurice Merleau-Ponty, fidèle en cela à l'une des lignes directrices tracées par Husserl au début du vingtième siècle, a suivi cette seconde voie, celle "de l'expérience et des données sensibles », et examine la manière dont la question de la légalité linguistique et de sa spécificité a pu se poser pour lui dans cette perspective. La référence au père de la linguistique, Ferdinand de Saussure, ayant jouée dans ce cadre une fonction cardinale, mais ambiguë, nous la prendrons comme point d'entrée dans ce problème. Comme nous allons le montrer, l'interprétation qu'en propose Merleau-Ponty est étroitement liée à sa conception des relations entre légalité perceptive et légalité linguistique.

\section{La question du langage en contexte phénoménologique. L'anti- idéalisme merleau-pontien}

Si la phénoménologie consiste, selon les mots de Husserl que Merleau-Ponty reprend, à amener «l'expérience (...) muette encore à l'expression pure de

${ }^{1}$ E. Husserl, Idées directrices pour une phénoménologie, tome premier, trad. fr. P. Ricoeur, Paris, Gallimard, [1913] 1950, p. 420-421.

${ }^{2}$ Ibid., p. 421 note (b). 
son propre sens $\gg{ }^{1}$, elle consiste d'abord, dans l'interprétation qu'en fait Merleau-Ponty, à « faire apparaître ${ }^{2}$ cette expérience muette, « la replacer sous notre regard, l'offrir à notre constatation $»^{3}$. Merleau-Ponty semble donc, contrairement au premier Husserl, privilégier la seconde voie, ce qui réserve a priori une portion congrue au problème de la grammaire pure et de la logique dans sa philosophie. En réalité - c'est évidemment crucial pour comprendre pourquoi Merleau-Ponty a quelque chose à nous dire de l'approche formelle des Recherches logiques - , les deux voies distinguées par Husserl ne forment pas une véritable alternative à ses yeux. Il s'en explique dans l'Avant-Propos de la Phénoménologie de la perception: selon lui, les essences ne peuvent être séparées de l'existence, le langage ne produisant à cet égard qu'un simulacre dont il ne faut pas être dupe.

Quels que puissent être les glissements de sens qui finalement nous ont livré le mot et le concept de conscience comme acquisition du langage, nous avons un moyen direct d'accéder à ce qu'il désigne, nous avons l'expérience de nous-mêmes, de cette conscience que nous sommes, c'est sur cette expérience que se mesurent toutes les significations du langage et c'est elle qui fait que justement le langage veut dire quelque chose pour nous. [...] Il ne faut donc pas dire avec J. Wahl que "Husserl sépare les essences de l'existence ». Les essences séparées sont celles du langage. C'est la fonction du langage de faire exister les essences dans une séparation qui, à vrai dire, n'est qu'apparente, puisque par lui elles reposent encore sur la vie antéprédicative de la conscience. Dans le silence de la conscience originaire, on voit apparaittre non seulement ce que veulent dire les mots, mais encore ce que veulent dire les choses, le noyau de signification primaire autour duquel s'organisent les actes de dénomination et d'expression ${ }^{4}$.

La séparation des essences par et dans le langage n'est donc, pour MerleauPonty, qu'apparente. Décrire, comme il pense que la philosophie doit le faire, « le rapport au monde, tel qu'il se prononce infatigablement en nous », c'est donc inséparablement faire apparaître « ce que veulent dire les mots » et «ce que veulent dire les choses ». Le risque d'une telle approche, cependant, serait d'identifier ces deux choses, ce qui réglerait abusivement l'un des problèmes soulevés par les éditeurs de ce dossier, celui du rapport entre la

${ }^{1}$ E. Husserl, Méditations cartésiennes, Paris, Colin, trad. fr. G. Peiffer et E. Levinas, 1931, p. 33 ; cité par M. Merleau-Ponty, Phénoménologie de la perception, Paris, Gallimard, [1945] 2009, p. 15. Désormais Php.

${ }^{2}$ Php, p. 16.

${ }^{3}$ Php, p. 18

${ }^{4}$ Ibid. Nous soulignons. 
légalité phénoménale et la légalité linguistique. Tel n'est pourtant pas ce que veut dire Merleau-Ponty, qui prend au contraire des précautions, dès la rédaction de La structure du comportement, pour les distinguer. Dès lors, ces formulations manifestent plutôt son souci d'éviter toute forme d'entente idéaliste de la signification linguistique et, par là même, de notre pensée, de notre réflexion, c'est-à-dire toute conception qui penserait le langage et la pensée comme étant séparés - c'est son terme - de notre vie réelle, qui sous-estimerait donc le fait que le langage est notre langage, la pensée notre pensée, et que cela conditionne qu'ils sont ce qu'ils sont. Si Merleau-Ponty a le souci, donc, de bien distinguer légalité linguistique et légalité phénoménale, il a également le souci de ne pas les séparer, et cela par intérêt pour ce qui fait l'unité de notre vie. Il formule par là même un défi : comment distinguer ces deux types de légalité sans être idéaliste ? C'est-à-dire sans sousestimer ou nier notre incarnation, le fait que le langage, la pensée, la réflexion sont le langage, la pensée et la réflexion d'homme dotés de corps?

Relativement au groupe de problèmes qui nous occupent dans ce dossier, la position de Merleau-Ponty est donc la suivante. Tout d'abord, il entreprend dans ses deux premiers ouvrages, la Structure du comportement et la Phénoménologie de la perception, d' " offrir à la constatation» du lecteur ce «rapport au monde, tel qu'il se prononce infatigablement en nous ${ }^{1}$. Rejoignant par ses moyens propres - la Gestalttheorie et les résultats de Kurt Goldstein - les travaux de Husserl sur la synthèse passive et l'intentionalité opérante, il découvre, littéralement, une structuration originaire du perçu. Mais il se trouve alors évidemment confronté au problème souligné dans la citation des Idées directrices par laquelle nous avons ouverte cet article : comment rendre compte philosophiquement, et donc par le langage, de cette structuration?

Comme il est bien connu, son ambition fut diplopique. Sa première préoccupation fut en effet de déjouer les pièges de l'empirisme, qui réduit la perception à une somme de sensations. Concevant de ce fait la structuration du perçu en termes de "signification », son souci fut cependant de ne pas verser dans le défaut - idéaliste - inverse, et donc de préserver la spécificité de l'ordre perceptif par rapport à l'ordre de la pensée, de la logique et du langage ${ }^{2}$. L'insistance sur la distinction entre la signification du perçu et la signification idéale (c'est-à-dire la signification du langage telle

\footnotetext{
${ }^{1}$ Php, p. 18.

${ }^{2}$ Les trois concepts se distinguent dans sa philosophie, mais certaines de ses critiques anti-idéalistes les mêlent dans un grand ensemble «intellectualiste »- ou y mêlent du moins leur entente la plus classique.
} 
que - selon Merleau-Ponty - on le conçoit classiquement) est ainsi une constante de son œuvre. Dès la Structure du comportement, il parle en effet, au sujet de la perception, de «significations qui ne sont pas de l'ordre logique $»^{1}$, qui sont, écrit-il encore dans les notes préparatoires au cours de 1953 sur «Le monde sensible et le monde de l'expression », des significations «non parlantes $»^{2}$. Dans la Phénoménologie de la perception, il affirme clairement qu'avec le sens d'une qualité sentie, il s'agit «d'une valeur expressive plutôt que d'une signification logique $»^{3}$. Dans les notes de travail reproduites dans Le visible et l'invisible, Merleau-Ponty insiste enfin : « il y a un monde du silence, le monde perçu, du moins, est un ordre où il y a des significations non langagières $»{ }^{4}$.

La situation se complique cependant du fait que, pour le phénoménologue français, l'idéalisme est un défaut auquel il faut se soustraire en matière perceptive, mais aussi, comme nous l'avons indiqué, en matière linguistique. Comme il l'explique au début du cours sur « Le monde sensible et le monde de l'expression ", à l'affirmation très nette de la différence du sens du perçu avec la signification linguistique idéale telle qu'on la conçoit traditionnellement ( «soumise, comme le résume Emmanuel de Saint Aubert, à une définition claire et distincte $»^{5}$ ), il adjoint le projet de prendre la mesure sur le plan du sens en général des exigences de la critique de l'idéalisme, révélées notamment lors de son étude de la perception, ce qui suppose en particulier de nuancer la distinction entre perception et langage, de faire en sorte, en tout cas, qu'elle ne dégénère pas en séparation.

La question des rapports entre le sens du perçu et la signification linguistique, et donc entre l'ordre du perçu et celui du langage se complique donc chez Merleau-Ponty de la réforme anti-idéaliste qu'il fait subir au concept de sens dans toute sa généralité. Or, le principal outil de cette réforme du sens du sens est le concept de "diacritique », dont on sait que Merleau-Ponty l'a emprunté, dans l'esprit sinon à la lettre, à Ferdinand de Saussure. Dans ce qui suit, nous allons analyser certaines des modalités de cet héritage, afin de mettre en évidence ce qu'elles révèlent de la relation que Merleau-Ponty entend penser entre légalité perceptive et légalité linguistique

\footnotetext{
${ }^{1}$ M. Merleau-Ponty, La structure du comportement, Paris, PUF, [1942] 2009, p. 135.

${ }^{2}$ M. Merleau-Ponty, Le monde sensible et le monde de l'expression. Cours au Collège de France, Notes, 1953, E. de Saint Aubert et S. Kristensen (éd.), Genève, MētisPresses, 2011, p. 50. Désormais MSME.

${ }^{3}$ Php, p. 28.

${ }^{4}$ M. Merleau-Ponty, Le visible et l'invisible. Suivi de Notes de travail, texte établi par C. Lefort, Paris, Gallimard, 1964, p. 223. Nous soulignons. Désormais VI.

${ }^{5}$ E. de Saint Aubert, « Conscience et expression », dans MSME, p. 17.
} 
— comme nous allons le voir, les difficultés que posent cet héritage sont aussi celles que pose cette relation.

\section{La critique de la «conception réflexive" du langage : le sens comme diacritique}

Au début de son cours de 1948 publié sous le titre Psychologie et pédagogie de l'enfant, le phénoménologue français critique une certaine entente du langage, sa « conception réflexive » ${ }^{1}$. Il entend ainsi dénoncer une conception du sens qui se trouve identifiée clairement au début du cours de 1953 : « Ceci [la notion de conscience] implique conception du sens comme essence $=$ ce qui répond à la question : quoi $?=$ définition. Toute conscience est saisie d'une essence de ce genre ou son application à un cas particulier. ${ }^{2}$ De manière fort significative, Merleau-Ponty fait alors référence à Husserl ${ }^{3}$, dont il critique dès 1951 la conception du langage, " original[e] et énigmatique ${ }^{4}$ ", mais surtout, en l'occurrence, excessivement idéaliste. Il commente en ces termes l'idée de grammaire universelle (telle qu'elle est exposée dans la quatrième des Recherches logiques) :

Ce projet suppose que le langage soit l'un des objets que la conscience constitue souverainement, les langues actuelles des cas très particuliers d'un langage possible dont elle détient le secret, - système de signes liés à leur signification par des rapports univoques et susceptibles, dans leur structure comme dans leur fonctionnement, d'une explicitation totale. Ainsi posé comme un objet devant la pensée, le langage ne saurait à son égard jouer d'autre rôle que celui d'accompagnateur, substitut, aide-mémoire ou moyen secondaire de communication ${ }^{5}$.

${ }^{1}$ M. Merleau-Ponty, Psychologie et pédagogie de l'enfant. Cours de Sorbonne, 1949-1952, J. Prunaire, Lagrasse (éd.), Verdier, 2001, p. 9. Nous nous référons à cet ouvrage par les initales $P P E$.

${ }^{2}$ MSME, p. 48.

${ }^{3}$ La citation précédente continue ainsi : «P. Ex. même les données hylétiques chez Husserl sont l'occasion d'une Auffassung als... qui est l'imposition d'une essence Husserl dit bien que toute constitution ne peut être de ce type, qu'il y a une Urkonstitution qui quitte les essences, mais ne la décrit guère ».

${ }^{4}$ M. Merleau-Ponty, «Sur la phénoménologie du langage », dans Signes, Paris, Gallimard, 1960, p. 136.

${ }^{5}$ Ibid., p. 137. 
Est ici ciblée toute conception pour laquelle le langage serait «posé comme un objet devant la pensée », et serait par elle constituée. On retrouve ici les caractéristiques de l'idéalisme dénoncées dans toute son œuvre: un sujet constituant, la conscience réflexive, pose devant lui un objet, qu'il peut en droit expliciter totalement, qui est donc à ses yeux transparent, clair, parfaitement adéquat à sa pensée, sans " puissance propre $»^{1}$.

Dans le domaine du langage, cette « conception réflexive » est donc hantée, selon les termes de La prose du monde, par le "fantôme d'un langage pur $»^{2}$. Et logiquement, les caractéristiques dénoncées sont celles-là mêmes qui étaient déjà en cause dans l'analyse de la perception dans la thèse de 1945 : rêve d'une signification " sans équivoque », d'un langage " absolument clair $»^{3}$, qui constituerait « seulement [un] code de "signaux" pour des idées $»^{4}$, et d'une adéquation telle entre la pensée et son expression que la seconde pourrait remplacer 《 sans reste $»^{5}$ la première sur la place publique, que la pensée serait, comme le dit Mauro Carbone, " dicible sans résidus »"

À la conception « réflexive » du langage identifiée par Merleau-Ponty est donc strictement corrélée une certaine conception de la conscience comme «constitu[ant] en toute clarté son objet»", lui étant tout à fait homogène, et formant donc une « conscience toute prête à être mise en mots, traduite en langage (...) déjà position d'un énoncé, conscience parlante $»^{8}$. Le sens possède la positivité dictée par ces conceptions : "essence » qui «par principe est claire », " unité rigoureuse », qui ne peut qu'être tout à fait ce qu'elle est ou n'être rien, sens parfaitement déterminé.

Là contre, Merleau-Ponty tient au contraire à penser dès 1945 que la parole, chez celui qui parle, «ne traduit pas une pensée déjà faite, mais l'accomplit ${ }^{9}$, et soutient donc que la pensée n'est pas autonome, qu'elle «n'est rien d'"intérieur", elle n'existe pas hors du monde et hors des

\footnotetext{
${ }^{1}$ PPE, p. 10.

${ }^{2}$ M. Merleau-Ponty, La prose du monde, Paris, Gallimard, 1969, p. 7. Désormais noté $P M$.

${ }^{3} P M$, p. 8.

${ }^{4}$ M. Merleau-Ponty, Recherches sur l'usage littéraire du langage. Cours au Collège de France. Notes, 1953, Genève, MētisPresses, 2013, p. 124.

${ }^{5} P M$, p. 180.

${ }^{6}$ M. Carbone, « Dicibilité du monde et historicité de vie. Expression, vérité, histoire dans la période intermédiaire de la pensée de Merleau-Ponty », dans La visibilité de l'invisible. Merleau-Ponty entre Césanne et Proust, Hildesheim, Olms, 2001, p. 60.

${ }^{7}$ MSME, p. 49.

${ }^{8}$ Ibid.

${ }^{9}$ Php, p. 217.
} 
mots $»{ }^{1}$. Or, Merleau-Ponty trouve pour ce faire dans la linguistique saussurienne - qui n'est bien sûr pas sa seule référence, mais joue un rôle crucial dans son argumentation - des ressources qui lui permettront de poser les bases de sa contre-attaque contre l'idéalisme, sur le plan du langage mais aussi sur celui de la perception.

La conception alternative du langage et du sens que Merleau-Ponty propose a été maintes fois commentée, et se trouve développée dans nombre de ses textes. Il y apparaît que, pour éloigner le «fantôme d'un langage pur », Merleau-Ponty va extraire de la linguistique structurale de Saussure de quoi introduire de la négativité dans le sens, ce mouvement conceptuel ayant pour emblème ce concept que Merleau-Ponty, après l'avoir découvert, ne cessera jamais d'employer : le «diacritique ». En 1952, il commence en ces termes le fameux article rédigé pour les Temps Modernes :

Ce que nous avons appris de Saussure, c'est que les signes un à un ne signifient rien, que chacun d'eux exprime moins un sens qu'il ne marque un écart de sens entre lui-même et les autres. Comme on peut en dire autant de ceux-ci, la langue est faite de différences sans termes, ou plus exactement les termes en elle ne sont engendrés que par les différences qui apparaissent entre eux $^{2}$.

Par rapport à la «conception réflexive» du langage, où la positivité de chaque signe, porteur d'une signification univoque, correspond à la positivité de chaque essence, la rupture est radicale, car ce que Merleau-Ponty extrait de Saussure, c'est l'idée que les signes n'ont pas de signification intrinsèque, qui leur serait accolée isolément, indépendamment des autres signes linguistiques, mais que chacun ne tient son sens que du fait qu'il est le signe qu'il est et non un autre, et donc que sa signification lui est fondamentalement conférée par le fait qu'il est différent des autres signes existants.

\footnotetext{
${ }^{1} P h p$, p. 223. L'《 intérieur » dont il est ici question est à comprendre comme ce qui s'oppose à l'extérieur, c'est-à-dire comme une intériorité étanche au sens de la seconde des Méditations métaphysiques.

${ }^{2}$ M. Merleau-Ponty, «Le langage indirect et les voix du silence », dans Signes, op. cit., p. 63. Nous soulignons. Il est fort intéressant de noter que dans le chapitre de $L a$ prose du monde intitulé "Le langage indirect », qui constitue une première version de ce texte, Merleau-Ponty n'employait pas le terme de « diacritique », qui n'apparaît que dans l'article rédigé pour Les Temps Modernes. Nous devons cette remarque à R. Kearney, «Écrire la chair. L'expression diacritique chez Merleau-Ponty », dans Chiasmi International, 2013, n¹5, p. 183.
} 
Comme Merleau-Ponty l'écrit dès 1949 dans son cours sur « La conscience et l'acquisition du langage $»$ :

La plus exacte caractéristique d'un mot est d'être « ce que les autres ne sont pas ». Il n'y a pas signification d'un mot, mais de tous les mots les uns par rapport aux autres (...). Ainsi le phénomène linguistique est cette coexistence d'une multiplicité de signes, qui, pris individuellement, n'ont pas de sens, mais qui se définissent à partir d'une totalité dont ils sont eux-mêmes les constituants ${ }^{1}$.

Une nouvelle conception de la signification procède de cette idée : la signification d'un signe, produit de l'écart, et même d'écarts (puisque chaque signe est différent de tous les autres signes du langage) ne peut recevoir aucune définition autonome, positive, pleinement déterminée, mais ne semble être déterminée que négativement, ou « comme "en creux" » ${ }^{2}$. L'importance du motif de l'écart apparaît encore lorsque, usant, abusant peut-être, du préfixe « inter », Merleau-Ponty écrit en 1952: «Le sens n'apparaît donc qu'à l'intersection et comme dans l'intervalle des mots $»^{3}$. Déclinant cette idée, Merleau-Ponty parle ainsi d'un " sens latéral ou oblique " ${ }^{4}$, de « signification latérale ou indirecte $»^{5}$ ou affirme " que tout langage est indirect ou allusif, est, si l'on veut, silence ${ }^{6}$. Et c'est donc cette idée fondamentale que Merleau-Ponty entend emprunter à Saussure lorsqu'il s'approprie son concept de "diacritique ", comme cela apparaît lorsqu'il écrit : "Saussure admet que la langue est essentiellement diacritique : les mots portent moins un sens qu'ils n'en écartent d'autres $\gg{ }^{7}$.

Emmanuel Alloa a montré à la suite de Richard Kearney que MerleauPonty donne par là une importance majeure à un concept a priori plutôt mineur de Saussure. Il exploite manifestement l'étymologie du terme "diacritique », comme étant ce «à travers quoi » (dia) une "distinction» (diakrisis) est faite, comme étant, comme la dernière citation l'indique,

${ }^{1} P P E$, p. 84.

${ }^{2} P P E$, p. 24. Merleau-Ponty fait ici référence à Jakobson.

${ }^{3}$ M. Merleau-Ponty, «Le langage indirect et les voix du silence», art. cit., p 68.

Nous soulignons.

${ }^{4}$ Ibid., p. 75.

${ }^{5}$ M. Merleau-Ponty, « Sur la phénoménologie du langage », art. cit., p. 122.

${ }^{6} \mathrm{M}$. Merleau-Ponty, « Le langage indirect et les voix du silence », art. cit., p. 70.

${ }^{7}$ PPE, p. 81. 
essentiellement ce qui «écarte»". L'allusion, le silence, l'oblique, la latéralité, le diacritique, tous ces concepts servent à marquer, par rapport à la pleine positivité présupposée par la conception réflexive, la relative négativité du sens du langage telle que Merleau-Ponty la découvre après Saussure.

La difficulté est que, si cette négativité sert fort pertinemment le projet métaphysique de Merleau-Ponty, elle ne semble pas totalement fidèle à la lettre du texte saussurien ; cela ne doit d'ailleurs pas nous étonner, nombre de commentateurs ayant déjà souligné la "liberté" de sa lecture du célèbre linguiste suisse ${ }^{2}$. Une certaine positivité du signe linguistique, corrélée chez Saussure à son caractère conventionnel, est ainsi délibérément ignorée par Merleau-Ponty. Or, il nous semble que, pour cette raison, notre auteur peine dans l'ensemble de son œuvre à distinguer les divers ordres de légalité perceptive et linguistique - qui l'intéressent. Ce qui motive son interprétation de Saussure mériterait donc pour cette raison un intérêt particulier. Pour le dire plus précisément, notre thèse est que l'appréciation singulière de l'œuvre saussurienne par Merleau-Ponty peut être rapportée au fait qu'il tient à penser une relation de continuité entre ces différents ordres, qu'il redoute, comme nous le rappelions, de les séparer, et que c'est à cause de cette préoccupation qu'il échouera in fine à distinguer différents types de légalité. Il ne peut s'agir ici de démontrer rigoureusement cette proposition; notre ambition, plus modeste, sera d'indiquer l'importance que revêt la lecture de Saussure pour notre question, et donc de mettre en évidence l'enjeu que représente, pour le lien entre structure du langage et forme du perçu, la question du caractère conventionnel du signe linguistique.

\section{Positivité sans conventionalité : les difficultés de la légalité perceptuelle}

Le point de départ de l'analyse est trivial: le concept saussurien de diacritique suppose des signes. À l'égard de cette nécessité, la position merleaupontienne semble être que tout, dans le perçu, fait potentiellement office de

${ }^{1}$ E. Alloa, «The Diacritical Nature of Meaning : Merleau-Ponty with Saussure », Chiasmi International, 2013, $\mathrm{n}^{\circ} 15$, p. 164-166.

${ }^{2}$ Voir par exemple le remarquable article de A.-P. Foultier, « Merleau-Ponty's Encounter with Saussure's Linguistics: Misreading, Reinterpretation or Prolongation? »(Chiasmi International, 2013, $\mathrm{n}^{\circ} 15$, p. 129-148) qui a le grand intérêt de synthétiser et d'ordonner les interprétations les plus connues de la relation de Merleau-Ponty à Saussure. 
signes : l'homme, parce qu'il est homme, et plus fondamentalement parce qu'il est vivant, donne du sens à ce qu'il perçoit - tout ce que nous percevons est de ce fait structuré et manifeste un sens.

La difficulté est que, comme cela est bien connu, les signes linguistiques sont, selon Saussure, conventionnels et que c'est à ce titre qu'ils bénéficient pour lui d'une forme de positivité que l'analyse merleaupontienne ne semble pas leur conférer. Saussure affirme en effet explicitement une forme de positivité du signe dans son Cours de linguistique générale:

Mais dire que tout est négatif dans la langue, cela n'est vrai que du signifié et du signifiant pris séparément : dès que l'on considère le signe dans sa totalité, on se trouve en présence d'une chose positive dans son ordre. (...) Bien que le signifié et le signifiant soient, chacun pris à part, purement différentiels et négatifs, leur combinaison est un fait positif ; c'est même la seule espèce de faits que comporte la langue, puisque le propre de l'institution linguistique est justement de maintenir le parallélisme entre ces deux ordres de différences ${ }^{1}$.

Notons-le: c'est l'institution linguistique qui, pour Saussure, maintient le parallélisme entre les deux ordres de différence, c'est elle qui assure, selon la formule employée par Jocelyn Benoist dans l'un des articles qu'il consacre à la question du structuralisme, «la mise en correspondance des deux séries $»^{2}$. Or, si, sur ces deux points (la positivité du signe et son caractère conventionnel), la position merleau-pontienne a manifestement oscillé, l'écart qu'il a toujours maintenu par rapport à Saussure est fort significatif.

En 1949 en effet, mû peut-être par l'enthousiasme suscité par la nouveauté, Merleau-Ponty semble affirmer le caractère entièrement négatif du langage. Il écrit ainsi : « Saussure admet que la langue est essentiellement diacritique : les mots portent moins un sens qu'ils n'en écartent d'autres. (...) Dans une langue, dit Saussure, tout est négatif, il n'y a que des différences sans termes positifs $»^{3}$. Du point de vue de la généralisation du concept de sens à la perception, la thèse est fort utile : nul besoin d'unités pour avoir des significations, des écarts suffisent. Mais des écarts entre quoi ? La notion d'écart est, par définition, relationnelle. Qu'est-ce qui permet de décider ce qui, dans l'infinie richesse du perçu mise en exergue par Merleau-Ponty lui-

${ }^{1}$ F. de Saussure, Cours de linguistique générale [1916], Paris, Payot, 1972, p. 166167.

${ }^{2}$ J. Benoist, « Le "dernier pas" du structuralisme : Lévi-Strauss et le dépassement du modèle linguistique », Philosophie, 2008, n 98 , p. 56.

${ }^{3} P P E$, p. 81. 
même, va jouer le rôle de signes, et donc les rapports à considérer? Le problème est que l'on ne voit guère comment l'on pourrait identifier des signes du perçu sans succomber à une forme de naturalisme, de détermination a priori du sensible. Mais si l'on n'en identifie pas, peut-on encore penser un sens du perçu? La «désidéalisation» merleau-pontienne du concept de sens, qui vise notamment à rendre pensable la relation entre légalité perceptive et légalité linguistique, doit éviter de perdre en chemin les termes qu'elle doit associer.

Dans l'article de 1952, prenant peut-être acte de cette difficulté, Merleau-Ponty exprime une thèse légèrement différente, qui tâche, semble-til, de rendre compte de la positivité du signe sans pour autant lui conférer aucun caractère a priori : " la langue, écrit-il ainsi, est faite de différences sans termes, ou plus exactement les termes en elle ne sont engendrés que par les différences qui apparaissent entre eux ${ }^{1}{ }^{1}$. Selon cette interprétation, il n'y aurait pas que des différences, mais des termes seraient engendrés par ces différences. Merleau-Ponty tenterait ainsi d'avoir la (relative) positivité du signe sans son caractère conventionnel.

Il est clair en effet que Merleau-Ponty refuse tout à fait la conventionalité du langage, comme il l'explicite lorsqu'il commente sa position en ces termes :

Il n'y a donc pas à la rigueur de signes conventionnels, simple notation d'une pensée pure et claire pour elle-même, il n'y a que des paroles dans lesquelles se contracte l'histoire de toute une langue, et qui accomplissent la communication sans aucune garantie, au milieu d'incroyables hasards linguistiques ${ }^{2}$.

Merleau-Ponty formule ici son interprétation de la célèbre thèse saussurienne du caractère arbitraire du signe, selon laquelle le signifiant « est immotivé, c'est-à-dire arbitraire par rapport au signifié, avec lequel il n'a aucune attache naturelle dans la réalité $\gg{ }^{3}$. Selon Saussure, cette thèse implique que

l'idée de « sœur » n'est liée par aucun rapport intérieur avec la suite de sons s-ö-r qui lui sert de signifiant ; il pourrait aussi bien être représenté par n'importe quelle autre : à preuve les différences entre les langues et l'existence

${ }^{1}$ M. Merleau-Ponty, «Le langage indirect et les voix du silence», art. cit., p. 63 (déjà cité). Nous soulignons.

${ }^{2}$ Php, p. 229.

3 F. de Saussure, Cours de linguistique générale, op. cit., p. 101. Ce qui est littéralement souligné est souligné par nous. 
même de langues différentes : le signifié « bœuf » a pour signifiant b-ö-f d'un côté de la frontière, et o-k-s (Ochs) de l'autre ${ }^{1}$.

Au contraire, précise-t-il, «tout moyen d'expression reçu dans une société repose en principe sur une habitude collective ou, ce qui revient au même, sur la convention $»^{2}$. Or, Merleau-Ponty critique clairement cette conception: le signifiant n'est certainement pas arbitraire par rapport au signifié, dans la mesure où selon lui le sens des mots est induit par les mots eux-mêmes, et donc où il $\mathrm{y}$ a bel et bien une relation essentielle entre ce sens et les mots qui le portent, et donc le signifié et le signifiant. De ce point de vue, concevoir des "signes conventionnels", ce serait régresser vers une conception idéaliste pour laquelle le sens d'un mot ne serait pas consubstantiellement le sens qu'il est parce qu'il est le sens de ce mot, et non d'un autre. L'ignorer, ce serait penser le sens indépendamment des signes qui les portent, qui apparaitraient alors, comme l'écrit nettement MerleauPonty, comme la «simple notation d'une pensée pure et claire pour ellemême ». Au contraire, il importe de rendre compte du fait que le sens des mots est induit par les mots eux-mêmes. C'est cette productivité sémiotique de la parole que le phénoménologue français semble donc vouloir dire lorsqu'il refuse, ou nuance fortement, la positivité du signe telle que Saussure la pense.

Plusieurs remarques s'imposent alors. Car il est frappant que la critique de la thèse saussurienne que Merleau-Ponty formule ici rejoigne pour partie la critique déjà formulée en 1939 par Émile Benveniste. Dans « Nature du signe linguistique ${ }^{3}$, Benveniste fait en effet apparaître que la thèse de Saussure est, telle que ce dernier la formule, inconséquente avec la consubstantialité du signifiant et du signifié qu'il affirme par ailleurs (notamment, remarque par exemple Benveniste, lorsqu'il compare la langue à une feuille de papier dont le recto serait la pensée et le verso serait le $\operatorname{son}^{4}$ ). L'unité du signe linguistique, telle que Saussure lui-même la définit, implique ainsi, soutient Benveniste, qu'

${ }_{2}^{1}$ Ibid., p. 100. Nous soulignons (en italique).

${ }^{2}$ Ibid., p. 100-101.

${ }^{3}$ É. Benveniste, « Nature du signe linguistique », Acta Linguistica, 1939, n ${ }^{\circ} 1$, p. $23-$ 29 ; repris dans Problèmes de linguistique générale, 1, Paris, Gallimard, 1966, p. 4955.

${ }^{4}$ Voir le Cours du linguistique générale, op. cit., p. 159 ; commenté par Benveniste dans « Nature du signe linguistique », art. cit., p. 52. 
[e]ntre le signifiant et le signifié, le lien n'est pas arbitraire ; au contraire, il est nécessaire. Le concept («signifié ») «bœuf» est forcément identique dans ma conscience à l'ensemble phonique (« signifiant») böf. Comment en serait-il autrement? Il y a entre eux symbiose si étroite que le concept « bœuf » est comme l'âme de l'image acoustique böf. L'esprit ne contient pas de formes vides, de concepts innomés ${ }^{1}$.

À lire Benveniste, nous pourrions donc penser qu'en critiquant l'arbitraire du signe, qui n'a de sens, précise-t-il, que «sous le regard impassible de Sirius $»^{2}$, Merleau-Ponty défende l'unité du signe linguistique contre l'interprétation idéalisante que certaines formules saussuriennes peuvent induire, et même clairement encourager. C'est d'ailleurs en ce sens que Merleau-Ponty semble lui-même comprendre Saussure lorsqu'il s'y réfère directement en Sorbonne : «Quand Saussure parle du caractère conventionnel du langage, il exprime dans un autre vocabulaire cette idée que le langage est "culturel", non "naturel" ".

Mais il est clair d'autre part - ce sera notre deuxième remarque que la critique merleau-pontienne de la thèse de l'arbitraire du signe excède la réforme de Benveniste. Il y a en effet un lien nécessaire, dit Benveniste, entre le signifiant et le signifié, au sens où le signifiant ne peut pas être le signifiant qu'il est sans être le signifiant de ce signifié-ci et non de celui-là. Mais, précise-t-il pourtant, il demeure quelque chose d'arbitraire dans la langue; simplement, il ne concerne pas, comme Saussure le disait, les différentes dimensions du signe, mais son lien à la réalité.

Ce qui est arbitraire, c'est que tel signe, et non tel autre, soit appliqué à tel élément de la réalité, et non à tel autre. En ce sens, et en ce sens seulement, il est permis de parler de contingence, et encore sera-ce moins pour donner au problème une solution que pour le signaler et en prendre provisoirement congé. [...] C'est en effet, transposé en termes linguistiques, le problème métaphysique de l'accord entre l'esprit et le monde, problème que le linguiste sera peut-être un jour en mesure d'aborder avec fruit, mais qu'il fera mieux pour l'instant de délaisser ${ }^{4}$.

Il y a un lien nécessaire, souligne Benveniste, entre tel signifié et tel signifiant car l'un et l'autre n'existent pour nous qu'ensemble. Pour autant, il n'est pas nécessaire, mais contingent, que ce soit tel couple signifié/signi-

${ }^{1}$ É. Benveniste, « Nature du signe linguistique », art. cit., p. 51.

2 Ibid.

${ }^{3}$ PPE, p. 82.

${ }^{4}$ É. Benveniste, « Nature du signe linguistique », art. cit., p. 52. 
fiant, et donc tel signe, qui serve dans telle langue à désigner tel élément de la réalité. Pour reprendre l'exemple développé au début de l'article, il n'y a pas de relation nécessaire entre "l'animal "bœuf" dans sa particularité concrète et "substantielle" »" et «böf d'une part, oks de l'autre »: mais la relation entre eux est de l'ordre de la «convention symbolique $»^{2}$. Il n'en demeure pas moins que cette relation contingente est alors présentée par Benveniste de manière fort prudente. Elle semble ici constituer pour lui plutôt le nom d'un problème - celui de l'accord métaphysique entre l'esprit et le monde - que celui d'une solution.

Or, c'est précisément ce problème que la conception de Merleau-Ponty entend traiter. En effet, Merleau-Ponty refuse la contingence, même mieux circonscrite, affirmée par Benveniste : il y a selon lui une relation essentielle entre les mots et leurs significations, mais aussi les réalités qu'ils désignent ${ }^{3}$. Il écrit ainsi dans la Phénoménologie de la perception que :

les mots, les voyelles, les phonèmes sont autant de manières de chanter le monde et qu'ils sont destinés à représenter les objets, non pas, comme le croyait la théorie naïve des onomatopées, en raison d'une ressemblance objective, mais parce qu'ils en extraient et au sens propre du mot en expriment l'essence émotionnelle ${ }^{4}$.

Les mots représentent les objets, leur relation avec tel ou tel élément de la réalité n'est pas purement contingente. Mais avec quel « élément de la réalité » chaque mot est-il en relation essentielle ? Non pas exactement, comme dans les hypothèses envisagées par Saussure et Benveniste, avec les objets eux-mêmes, mais avec leur «essence émotionnelle »: il y a une relation nécessaire entre «l'essence émotionnelle » d'un objet et « le sens émotionnel du mot » qui le désigne, pour la raison que c'est l'émotion, le projet moteur, existentiel, qui fait de l'un et de l'autre les sens qu'ils sont, et cela parce qu'ils l'« expriment» l'un et l'autre.

Il est alors fascinant de constater que Merleau-Ponty propose ici une conception dont il va lui-même critiquer ultérieurement une forme d'insuffisance et de naïveté, mais qui, engage aussi, positivement, certaines de ses positions métaphysiques les plus personnelles. Sa source, en effet, se trouve dans le souci merleau-pontien de l'unité de notre vie; mais de ce fait, la

${ }^{1}$ Ibid., p. 50.

${ }^{2}$ Ibid., p. 53.

${ }^{3}$ En réalité, nous esquisserons cette idée, le cœur de la thèse merleau-pontienne consiste à contester cette différence.

${ }^{4}$ Php, p. 228. 
reconnaissance des différents niveaux d'organisation dans les phénomènes humains - et en particulier, en l'occurrence, celle d'un niveau d'organisation, conventionnelle, propre au langage - est rendue difficile. De ce point de vue, le règne de l'écart semble être aussi celui de la confusion des différents types de structures.

Ainsi, l'un des aspects de la conception «émotiviste » est que l'homme ne distingue dans la réalité tel élément de tel autre, c'est-à-dire qu'il n'y a pour lui tel monde structuré de telle manière et non de telle autre, et donc tel monde, que parce que ce qu'il perçoit suscite en lui telle ou telle émotion, a pour lui telle ou telle « signification motrice». C'est donc cette émotion, cette « signification motrice » qui structure le monde qu'il perçoit. La conséquence, Merleau-Ponty l'assume ici tout à fait : au monde, l'homme superpose toujours et inévitablement, par la « gesticulation émotionnelle ${ }^{1}$ qui caractérise sa vie, «le monde selon l'homme $»^{2}$. La thèse, ainsi exprimée, est d'une grande radicalité : si les émotions diffèrent, le monde perçu doit différer aussi, et l'idée même d'une nature humaine commune indépendamment de la culture, mais aussi des émotions des uns et des autres devient problématique ${ }^{3}$. Pour l'homme qui la perçoit, il n'existe donc aucune réalité indépendante de la signification qu'elle prend pour lui - le partage entre réalité et signification se trouve totalement contesté, le « privilège de la Raison $\gg{ }^{4}$ devient fort problématique.

La difficulté se situe en ce point précis. Car cela implique une chose essentielle du point de vue linguistique : le langage ne peut plus désormais faire référence à autre chose qu'au «monde selon l'homme». La question à laquelle Merleau-Ponty se trouve ensuite confrontée parait inéluctable: comment rendre compte de la généralité de la pensée à partir de cette signification linguistique émotionnelle ? Comme le remarque Renaud Barbaras,

on se demande comment une telle dérivation est possible, comment un sens émotionnel pourra jamais s'égaler à un sens conceptuel. [...] Que le geste

${ }^{1}$ Php, p. 229.

2 Merleau-Ponty écrit exactement: "Il faudrait donc chercher les premières ébauches du langage dans la gesticulation émotionnelle par laquelle l'homme superpose au monde donné le monde selon l'homme. »

${ }^{3} \mathrm{~L}$ '《 idiosyncrasie » des « mondes anthropologiques » dans lesquels nous enferment les analyses de La phénoménologie de la perception se trouve mise en évidence par É. Bimbenet, dans les deux dernières sous-parties du chapitre 2 de Nature et humanité: le problème anthropologique dans l'œuvre de Merleau-Ponty, Paris, Vrin, 2004.

${ }^{4}$ Php, p. 231. 
silencieux ne soit pas lié à la nature sur un mode causal ne permet pas de comprendre qu'il se dépasse vers une signification linguistique ${ }^{1}$.

Pour le dire en un mot, l'identification merleau-pontienne du monde et du «monde selon l'homme» pose le problème de la vérité. Que ce concept précis fasse problème, l'auteur lui-même le reconnaît du reste dès 1947, dans cette note célèbre de «La métaphysique dans l'homme» où, pour la première fois, Merleau-Ponty parle du projet qui l'occupera jusqu'à son décès :

Il y aurait évidemment lieu de décrire précisément le passage de la foi perceptive à la vérité explicite telle qu'on la rencontre au niveau du langage, du concept et du monde culturel. Nous comptons le faire dans un travail consacré à l'Origine de la vérité ${ }^{2}$.

Il s'agirait bien sûr de poursuivre cette étude et d'étudier les péripéties de cette quête. Dans le cadre de cet article, nous souhaiterions insister sur un point: si l'on veut reconnaître une forme au perçu et une structure au langage, et penser la seconde en lien avec la première, comme le fait inévitablement la phénoménologie dès lors qu'elle choisit la voie «de l'expérience et des données sensibles », il importe de se garder d'un écueil : l'écrasement de la généralité de la pensée. Que pour s'en préserver, il faille reconnaître une positivité, conventionnelle ou non, propre au langage, voilà ce que l'analyse de la lecture merleau-pontienne de Saussure nous incline à penser.

\section{Conclusion}

Nous sommes partie de l'idée que, lorsqu'il entreprit d'analyser la structuration du perçu, Merleau-Ponty a taché d'éviter toute pensée idéaliste de cette forme et a pour cela trouvé des ressources dans le concept saussurien, ou d'origine saussurienne, de diacritique, qui constitue la deuxième version de la Gestalt merleau-pontienne. Le problème est que, ayant emprunté à Saussure de quoi repenser l'ensemble de l'univers du sens, et donc la forme du perçu comme la structure du langage, il semble avoir laissé de côté ce qui

\footnotetext{
${ }^{1}$ R. Barbaras, « De la parole à l'être », dans Le tournant de l'expérience. Recherches sur la philosophie de Merleau-Ponty, Paris, Vrin, 1998, p. 189-190.

${ }^{2}$ M. Merleau-Ponty, «Le métaphysique dans l'homme », Sens et non-sens, Paris, Gallimard, 1996, p. 115 note 1.
} 
chez Saussure lui semblait pouvoir constituer le ferment d'une séparation entre eux. La conventionalité du signe et par là même une certaine manière de penser la positivité du langage ont ainsi été critiquées par Merleau-Ponty, au profit d'une pensée de l'enracinement de la structure linguistique dans la forme du perçu et, de ce fait, d'une certaine homogénéisation de leurs régimes de légalité respectives. Le prix en est, nous semble-t-il, une difficulté à penser la vérité et la généralité du sens. Cela apparaît clairement dans la Phénoménologie de la perception et - il faudrait évidemment le prouver il ne semble pas que Merleau-Ponty règle le problème par la suite ${ }^{1}$. Le refus merleau-pontien de penser la séparation de la forme du perçu et de la structure du langage, qui peut être rapporté à sa recherche de l'originaire, à son souci transcendantal, et qui se traduit dans sa singulière lecture de Saussure semble donc avoir pour conséquence une certaine mutilation, dans sa philosophie, de la légalité propre au langage.

${ }^{1}$ Pour une démonstration, nous invitons le lecteur à se référer à la dernière partie de notre thèse de doctorat, déjà citée, et en particulier à son chapitre 7 . 